\title{
Molecular diagnostic technologies and end user applications: potentials and challenges
}

\author{
Yeshitila Degefu
}

MTT Agrifood Research Finland, Biotechnology and Food Research, Seed Potato Biotechnology, Tutkimusasemantie 15, 92400, Ruukki, Finland, yeshitila.degefu@mtt.fi

\begin{abstract}
Conventional methods of pathogen identification have often depended on identification of disease symptoms, isolation and culturing of the organisms, and identification by morphology and biochemical tests. The major limitations of these culture based morphological approaches, however, are the reliance on the ability of the organism to be cultured, the time consuming nature and requirement of extensive taxonomic expertise. Furthermore, diagnosis of plant diseases can be even more difficult with asymptomatically infected propagative materials such as tree grafting stocks or potato tubers. The use of molecular methods can circumvent many of these shortcomings. Accordingly, there have been significant developments in the area of molecular detection of plant pathogens in the last three decades. The advent of antibody based detection, the monoclonal antibodies and the enzyme linked Immunosorbent assay (ELISA), was an important turning point in virology and bacteriology. Then came the DNA based technologies, such as the polymerase chain reaction (PCR) which revolutionised molecular diagnostics and biological sciences. In the last decade the range of targets that can be diagnosed using diagnostic PCR have grown tremendously. The very flexibility and application specific variations in the basic theme of the system have allowed the development of many PCR variants adapted to wide range of applications. Furthermore, diagnostic PCR has been greatly improved by the introduction of the second generation PCR, known as the Real time PCR where closed-tube fluorescence detection and quantification during PCR amplification (in real time) is possible eliminating the need for laborious post-PCR sample processing steps which greatly reduces the risk of carryover contamination. Using Real Time PCR, it is possible not only to detect the presence or absence of the target pathogen, but it is also possible to quantify the amount present in the sample allowing the quantitative assessment of the number of the pathogen in the sample. Enumerating the pathogen upon detection is crucial to estimate the potential risks with respect to diseases development and provides a useful basis for diseases management decisions. Crops can be attacked by many pathogens which, in addition, often occur in complexes. Therefore, many disease diagnostic applications require simultaneous detection and quantification of several targets. Methodological limitations, however, are in many cases the reason for developing simplex or assays designed for only few targets. The DNA Microarray technology, originally designed to study gene expression and generate single nucleotide polymorphism (SNP) profiles, is currently a new and emerging pathogen diagnostic technology, which in theory, offers a platform for unlimited multiplexing capability. It is viewed as a technology that fundamentally alter molecular diagnostics. The fast growing databases generated by genomics and biosystematics research provides unique opportunity for the design of more versatile, high-throughput, sensitive and specific molecular assays which will address the major limitations of the current technologies and benefit plant pathology.
\end{abstract}

Key words: Molecular diagnostics, Pathogen detection, Real time PCR, Microarray, Diagnostic PCR 


\section{Introduction}

Accurate identification and early detection of pathogens is a crucial step in health care, agriculture and environmental monitoring including the fight against bioterrorism (Ivnistski, et al., 2003, Schaad and Frederick, 2002, Schaad et al., 2003) plant disease management. The failure to adequately identify and detect plant pathogens using conventional, culture based morphological techniques has led to the development of nucleic acid based molecular approaches (Lievens et al., 2005, López et al 2003). Molecular diagnostic began to develop a real momentum after the introduction of polymerase chain reaction (PCR) in the mid 1980s and the first PCR based detection of a pathogen in diseased plants was published in the beginning of 1990s (Rasmussen \& Wulff, 1991). To date an increasing number of diagnostic laboratories is adapting molecular methods for routine detection of pathogens. With the advances in molecular biology and biosystematics, the techniques available have evolved significantly in the last decade, and besides conventional PCR other technologically advanced methodologies such as the second generation PCR known as the real time PCR and microarrays which allows unlimited multiplexing capability have the potential to bring pathogen detection to a new and improved level of efficiency and reliability (Mumford et al., 2006).

However, while the specificity and sensitivity of detection of pathogens are greatly improved and pathogen detection is becoming simpler and faster, there are still major challenges, technical and economic nature, which need to be addressed to ensure the emergence of reliable detection system for routine applications. This short review evaluates the major technical demands that must be met and how the existing and emerging technologies are developing to fulfil these demands and what limitations still exist. In developing a tool for pathogen detection, issues such as detection specificity and sensitivity are very important. In addition multiplexing, quantification and cost effectiveness are increasingly becoming important features of a diagnostic technology. There is also a growing need for a field deployable portable rapid detection system that provides the capability for pathogen testing and identification in the field. These major required features are summarized below.

\section{Detection specificity and sensitivity}

Sensitivity and specificity are numeric measures of effectiveness of a detection system Peruski \& Peruski, 2003, Malorny et al., 2003). Diagnostic specificity is defined as a measure of the degree to which the method is affected by non target components present in a sample, which may result in false positive responses. Diagnostic sensitivity is defined as a measure of the degree to detect the target pathogen in the sample, which may result in false negative responses (Malorny et al., 2003). Too low sensitivity often leads to false negatives. Thus, a high degree of diagnostic accuracy is characterized by the ability to detect, true and precisely the target micro organism from a sample without interference from non target components. The high degree of sensitivity of molecular methods made presymptomatic detection and quantification of pathogens possible.

One of the most important advantages that molecular based detection has over conventional diagnostic detection methods is the high specificity. That is the ability to distinguish closely related organisms. The specificity of PCR, be it conventional or real-time, depends upon the designing of proper PCR primers that are unique to the target organism. Highly conserved gene regions are often the target for designing primers. Closely related microbial species often differ in a single (single-nucleotide polymorphism (SNPs) to few bases in such genes. PCR allows detection of such SNPs (Papp et al., 2003). With the advancements in high throughput DNA sequencing more and more genomes of plant pathogens are sequenced and nucleotide sequence data will be available increasing the possibility for designing unique primers and probes for specific detection of pathogens.

PCR is a highly sensitive technology. However, its sensitivity is greatly affected by the presence of inhibitors which prevent or reduce amplification. A wide range of inhibitors are reported (for detailed reviews Wilson, 1997; Yang \& Rothman, 2004 and references therein). Although their mode of action is not clear, these inhibitors are believed to interfere with the polymerase activity for amplification of the target DNA. On the other hand, it is worth mentioning that the high sensitivity of PCR also causes one of the limitations of PCR, that is detection sensitivity exceeding threshold levels or clinical significance and false positive results from slight DNA contamination (Yang \& Rothman, 2004). Hence, stringent conditions are 
necessary in conducting the assay and proper negative controls must be included in the test. It is also recommended to have separate dedicated areas for pre- and post PCR handling.

\section{Determination of viability}

Nucleic acid based detection methods currently applied in pathogen detection are based on nucleic acid hybridization or PCR. These methods can be designed to detect either DNA or mRNA. Whereas DNA based detection method is often more straightforward than that of mRNA, the stability of DNA leads to the possibility that DNA based methods yield positive results from non-viable or dead pathogens. One of the main goals of pathogen detection system, besides determining the presence and absence of the pathogen, is the viability since in the event of positive result it is important to know whether the pathogen detected poses threat to crop production, public health or food safety. The lack of discriminating viable from dead cells is a pitfall common to the nucleic acid based detection systems including microarrays and diagnostic PCR (Call, 2005, Keer \& Birch, 2003). Artz et al (2006) demonstrated that prolonged detection of non viable cells led to potential overestimation in the quantitative real time detection of Escherchia coli.

In order to circumvent this problem many studies consider enrichment culturing (BIO PCR) instead of direct PCR. While the system allows the detection of only viable cells and helps in elimination of possible PCR inhibitors, it is not appropriate approach for quantitative assay. Therefore, the lack of ability to distinguish between viable and dead cells, and the lack of sample preparation methods that do not involve enrichment culturing are currently limiting the implementation of quantitative PCR for routine diagnostic use. Molecular methods for inferring pathogen viability focus on detecting mRNA in a sample as mRNA species are believed to be labile with a very short half life (seconds to minutes) after cell death. However, although it is in theory a more accurate indicator of viable micro organisms there has been report of poor correlation between the two variables (Sung et al. 2004). More work is still needed to verify the question.

\section{Pathogen quantification}

Although new, rapid detection and identification technologies are becoming available for various pathogens, pathogen quantification remains to be one of the main challenges in the disease management of many crops. Quantification of a pathogen upon its detection and identification is an important aspect as it can be used to estimate its potential risk regarding disease development, establishment and spread of inoculum and economic loss. In addition it provides information for well informed disease management decisions. PCR is ideal for detection of small amount of the target but one of its limitations has been quantification. Three PCR variants namely limiting dilution PCR, kinetic PCR and competitive PCR (Hermansson \& Lindgren, 2001 and reference therein) have been used for quantitative analysis of DNA. However, all are based on end point measurements of the amount of DNA produced which makes estimation of initial concentration of DNA and quantification rather problematic. Even the emerging microarray technology has limitations with respect to microbial quantification in complex environmental samples due to the fact that microarray hybridization signals could vary depending on target abundance and hybridization efficiency (Wu et al. 2001). In other words, a low abundance target with high genetic similarity to a microarray probe might produce a stronger hybridization signal compared with a higher abundance target that has low similarity to the same microarray probe. Slide to slide variations from a particular probe, same hybridization condition and amount of DNA was also reported (White et al. 2005) leading to a speculation that variation from the printing of probes from slide to slide may contribute to such differences. On the other hand, efforts are underway towards adding a quantitative aspect in the array technology (Lievens, et al. 2005; Rudi et al. 2003)

Due to the advancement of fluorogenic chemistry, a second generation PCR known as real time PCR has become an emerging technique for the detection and quantification of micro organisms in the environment. In PCR the target DNA sequence is amplified over a number of denaturation-annealingextension cycles. In a conventional PCR, only the final concentration of the amplicons may be monitored using a DNA binding fluorescent dye. However, in the quantitative real time PCR, the concentration of the amplicons is monitored throughout the amplification cycles using a group of fluorescent reagents. The fluorescence intensity emitted during this process reflects the amplicons concentration in real time. Undoubtedly most of the future tests will be quantitative in nature and the real time detection system will be 
a method of choice. The real time data will serve as useful basis for establishing inoculum threshold levels and detailed analysis of disease epidemics.

\section{Multiplexing}

Crops can be infected by numerous pathogens and they may be present in plants in complexes. Therefore, it is desirable to develop technology that can detect multiple pathogens simultaneously. The methodological limitations however, are in many cases the reasons for developing simplex or assays only including few targets. Multiplex PCR, a PCR variant which is designed to amplify multiple targets by using multiple primer sets in the same reaction, has been applied in many tests. Multiplex PCR assays can be tedious and time consuming to establish requiring lengthy optimization processes (Elnifro et al. 2000). Among the drawbacks of such variant PCR assays are that the sensitivity is decreased enormously and the number of different targets to be amplified in one assay is limited (Bamaga et al. 2003). Moreover, the dynamic range of the target present in the sample to be tested is not always reflected in the outcome of the test. That is targets that are present in very low amounts will most of the time not amplified in contrast to those that are present abundantly.

The real-time PCR offer better multiplexing possibilities, however, multiplexing is still limited by the availability of dyes emitting fluorescence at different wavelengths. Thus, detection of more than few pathogens is currently not possible using these systems.

The DNA microarray technology originally designed to study gene expression and generate single nucleotide polymorphism (SNP) profiles, is currently a new and emerging pathogen diagnostic technology which in theory, offers a platform for unlimited multiplexing capability. The principle of microarray is the hybridization of fluorescently labelled sequences or targets to their complementary sequences spotted on solid surface, such as glass slides, serving as probes. Tens of thousands of such DNA probes can be spotted in a defined and addressable configuration on the glass slide forming the chip. The unlimited capability for simultaneous detection of pathogens makes microarrays to be an approach with a potential capacity of detecting all relevant pathogens of a specific crop. Development of microarrays for diagnostic applications is a recent history. In plant pathology the method was applied for identifying oomycete, nematode bacterial and fugal DNA from pure cultures (Fessahaie et al. 2003; Lévesque et al. 1998; Lievens, et al. 2003; Uehara et al., 1999). However, for application in practice, pathogens should be detected from environmental samples (plants, soil, etc.). Recently the possibilities of parallel detection of pathogens from such environments were shown (Lievens et al. 2003; Lievens, et al. 2004; Nicolaison, et al. 2005). In contrast with studies using pure cultures, microarray-mediated analysis from environmental samples presents several challenges that must be addressed (Whittle, et al. 2005; Zhou and Thompson, 2002)

\section{Conclusions and prospects}

Currently more and more diagnostic laboratories and inspection agencies are using molecular methods for detection and identification of pathogens. The development of more versatile robust and cost effective systems, allowing for greater sensitivity and specificity, elevated throughput and detection of multiple microbes will continue over the coming years. Pathogen detection is only the first step; quantification and isolate characterization are crucial elements in diagnostics. Diagnostic technology is moving from qualitative to quantitative and there is no doubt that most tests will be quantitative in the future. Microarray-based technology is the most suitable technique for multiple pathogen detection in a single assay. Currently microarrays can be expensive for routine application. However, with reducing fabrication costs, the cost per sample will be significantly lower. The effort to add a quantitative aspect to microarrays must continue and more work is needed to address the challenges of working on environmental samples where contaminants (humic matter, organic substances, heavy metals etc) may interfere with DNA hybridization and affect the performance of microarrays.

Adding innovative molecular tools for differentiating viable from non-viable organisms should be given emphasis in developing diagnostic assays. 


\section{References}

Artz, R.R.E., Avery, L.M., Jones, D.L. \& Killham, K. 2006. Potential pitfalls in the quantitative molecular detection of Eschrichia coli 0157:H7 in environmental matrices. Can. J. Microbiol 52:482-488.

Bamaga, M.S., Wright, D.J. \& Zhang, H. 2003. An assessment of multiplex PCR assays for differentiating clinically important mycobacteria based on pncA gene variation. Mol. Cell. Probes 17: 69-75.

Call, D.R. Challenges and opportunities for pathogen detection using DNA microarrays. Critical Rev. Microbiol 31: 91-99.

Elnifro, E.M., Ashishi, A.M., Cooper, R.J. \& Klapper, P.E. 2000. Multiplex PCR: Optimization and application in diagnostic virology. Clin. Microbiol.Rev. 13: 559-570.

Fessehaie, A., De Boer, S.H. \& Lévesque, C.A. 2003. An Oligonucleotide array for the identification and differentiation of bacteria pathogenic on potato. Phytopathology 93: 262-269

Hermansson, A. \& Lindgren, P-E. 2001. Quantification of Ammonia-oxidizing bacteria in arable soil by real-time PCR. Appl. Envion. Microbiol. 67: 972-976.

Ivnitski, D., O’Neil, D. J., Gattuso, A., Schlicht, R., Calidonna, M. \& Fisher, R. 2003. Nucleic acid approaches for detection and identification of biological warfare and infectious disease agents. BioTechniques 35:862-869.

Keer, J.T. \& Bircéh, L. 2003. Molecular methods for assessment of bacterial viability. J.Microbiol. Methods 53:175183.

Lievens, B., Bart, P. \& Thomma, P.H.J. 2005. Recent developments in pathogen detection arrays: Implications for fungal plant pathogens and use in practice. Phytopathol. 95: 1374-1380.

Lievens, B., Brouer, M., Vanachter, A.C.R.C., Lévesque C.A., Cammue, B.P.A. \& Thomma, B.P.H.J. 2003. Quantitative assessment of phytopathogenic fungi in various substrates using DNA macroarray. Environ. Microbiol. 7: 1698-1710

Lievens, B., Hanssen, I.R.M., Vanachter, A.C.R.C., Cammue, B.P.A. \& Thomma, B.P.H.J. 2004. Root and foot rot on tomato caused by Phytophtora infestans detected in Belgium. Plant Dis. 88:86.

Lévesque, C.A., Harlton, C.E. \& de Cock, A.W.A.M. 1998. Identification of some oomycetes by reverse dot blot hybridization. Phytopathol.88: 213-222.

López, M.L., Bertolini, E. Olmos, A., Caruso, P., Goris, M.T., Llop, P., Penyalver, R. \& Cambra, M. 2003. Innovative tools for detection of plant pathogenic viruses and bacteria. Int. Microbiol. 6:233-243.

Malorny, B., Tassios, P.T., Rådström, Cook, N., Wagner, M. \& Hoorfar, J. 2003. Standardization of diagnostic PCR for the detection of foodborne pathogens. Int.J. Food Microbiol. 83:39-48.

Mumford, R., Boonham, N., Tolinson, J. \& Barker, I. 2006. Advances in molecular phytodiagnostics- new solutions for old problems. European J. Plant Pathol.116: 1-19.

Nicolaison, M., Justesen, A.F., Thrane, U., Skouboe, P. \& Holmstrom, K. 2005. An oligonucleotide microarray for identification and differentiation of trichothecene producing and non-producing Fusarium species occurring on cereal grain. J. Microbiol. Methods 62: 57-69.

Papp, A.C., Pinsonneault., J.K., Cooke, G. \& Sadee, W. 2003. Single nucleotide polymorphism genotyping using allele-specific PCR and fluorescence melting curves. BioTechniques 34:1068-1072.

Peruski, L.F. \& Peruski A.H. 2003. Rapid diagnostic assays in the genomic biology era: detection and identification of infectious disease and biological weapon agents. BioTechniques 35: 840-846.

Rasmussen, O.F. \& Wulff, B.S. 1991. Detection of Pseudomonas syringae pv pisi using PCR . In Proceedings $4^{\text {th }}$ International working group on Pseudomonas syringae pathovars. KLuwer Academic Publishers, Dordrecht, The Netherlands. PP 369-376.

Rudi, K., Rud, I. \& Holck, A. 2003. A novel multiplex quantitative DNA array based PCR (MQDA-PCR) for quantification of transgenic maize in food and feed. Nucleic Acids Res. 31: e62

Schaad, N.W. \& Frederick, R.D. 2002. Real-time PCR and its application for rapid plant disease diagnostics. Can. J. Plant Pathol. 24: 250-258

Schaad, N.W., Frederick, R.D., Shaw J., Schneider, W.L., Hickson, R., Petrillo, M.D. \& Luster, D.G. 2003. Advances in molecular-based diagnostics in meeting crop biosecurity and phytosanitory issues. Ann. Rev. Phytopathol. 41: 305-324.

Sung, K.D., Stern, N.J. \& Hiett, K.L. 2004. Relationship of messenger RNA Reverse Transcriptase- Polymerase Chain Reaction signal to Campylobacter spp. viability. Avian Dis. 48:254-262.

Uehara, T., Kushida, A. \& Momota, Y. 1999. Rapid and sensitive identification of Pratylenchus spp. using reverse dot blot hybridization. Nematology 1: 549-555.

Wu., L., Thompson, D.K., Li, G., Hurt, R.A., Tiedje, J.M. \& Zhou, J. 2001. Development and evaluation of functional gene array for detection of selected genes in the environment. Appl. Environ. Microbiol. 67:5780-5790.

Whittle, I.H.F., Klammer, S.H. \& Insam, H. 2005. Design and application of an oligonucleotide microarray for investigation of compost microbial communities. J.Microbiol. Methods 62: 37-56. 
Yang, S. \& Rothman, R.E. 2004. PCR-based diagnostics for infectious diseases: uses, limitations, and future applications in acute-care settings. THE LANCET Infectious Dis. 4: 337-348.

Zhou, J. \& Thompson, D.K. 2002. Challenges in applying microarrays to environmental studies . Curr. Opinions Biotechnol. 13:204-204. 